\title{
Optimal procedures for home visits - A case study
}

Filipe Alves, Florbela P. Fernandes, Ana I. Pereira, and Adília Fernandes

Citation: AIP Conference Proceedings 1863, 270002 (2017);

View online: https://doi.org/10.1063/1.4992424

View Table of Contents: http://aip.scitation.org/toc/apc/1863/1

Published by the American Institute of Physics 


\title{
Optimal Procedures for Home Visits — a Case Study
}

\author{
Filipe Alves*, Florbela P. Fernandes*, Ana I. Pereira* ${ }^{* \dagger}$ and Adília Fernandes* \\ * Polytechnic Institute of Bragança, Portugal \\ ${ }^{\dagger}$ AL GORITMI,University of Minho, Portugal
}

\begin{abstract}
In Portugal the population is ageing. Therefore, the provision of health care at patients' home is becoming an important social and health area; this health service is provided by professional teams (usually composed by nurses) of the Health Centers. Nowadays, the scheduling of the visits is made manually. The proposal of this work is to do the scheduling automatically in order to minimize the overall time spent by the professional teams in the visiting activity. In this work the genetic algorithm was used to solve the optimization problem. Some numerical results are presented.
\end{abstract}

Keywords: Genetic Algorithm. Health Care. Optimization.

PACS: $02.60 . \mathrm{Pn}$

\section{INTRODUCTION}

According to the World Health Organization the ageing people and dependency rate care of older people in Europe, namely in Portugal, is increasing. The National Health System has to deal with more and more demanding scenarios in what concerns home visits. The provision of home health care is playing an increasingly important role in Europe, as it is economically advantageous to keep people at home instead of providing them a hospital bed [1].

The home-based care provided by public or private entities has been the subject of recent research mainly in the operations research area with particular attention on route's optimization and on the staff teams composition that provide this kind of services $[1,2,3,4]$.

To provide home-based care, Health Centers play a very important role since they are closer to the population. In this context, Health Centers have to organize and schedule the professional teams. This represents a complex problem being its main goal to minimize the time needed to do all the visits.

The paper is organized as follows: first, it is given a description of the problem is stated with its mathematical formulation; then a small summary of the genetic algorithm method (GA) since it was the method chosen to solve the problem; some results are presented and a comparison is made with another version of GA method. Finally some conclusions and future work ideas are given.

\section{MATHEMATICAL MODELLING OF THE PROBLEM}

Health Centers have nurses devoted partially to the task of providing health care at home patients, who live at any location in the area under supervision of the Health Center. The main objective is to minimize the total time needed to do all these visits.

In this section the mathematical modelling of the optimization problem is presented: how to schedule the home visits in order to minimize all the time needed.

So, it should be considered the total number of nurses that do the home visits, $N N$; the total number of patients that need some treatments at their home, $N P$; and all different locations, $N L$. It is also necessary the description of all treatments provided by each nurse (not all nurses perform all treatments), the time between the locations, the treatments that each patient should do (can be more that one) and the time needed to do each treatment.

Consider variable $x=\left(p_{1}, \ldots, p_{N P} ; n_{1}, \ldots, n_{N P}\right)$, where the patient $p_{i}$ will be visited by the nurse $n_{i}, i=1, \ldots, N P$. For a given $x$ is possible to define the nurse schedule, $S(l), l=1, \ldots, N N$. The objective function is defined as

$$
f(x)=\max _{l=1, \ldots, N N} S(l)
$$

which represents the maximum time needed by the last nurse to finish her last treatment to her last patient, counting even with the return journey to the starting point (Health Center). And the constrained optimization problem will be 
defined as

$$
\min f(x)
$$

where $x=\left(p_{1}, \ldots, p_{N P} ; n_{1}, \ldots, n_{N P}\right)$ with $p_{i} \in\{1, \ldots, N P\}$ and $n_{i} \in\{1, \ldots, N N\} ; p_{i} \neq p_{j}$ for $\forall i \neq j$; and all $n_{i}$ need to do all treatments of the patient $p_{i}$ for $i=1, \ldots, N P$.

\section{Case Study}

In the present case study, the Health Center is located at Bragança District (Location 1) and has three nurses assigned to perform four different treatments, with different average times, as described in Table 1.

TABLE 1. Average time ( $\mathrm{min}$ ) of treatments that each nurse performs in the case study.

\begin{tabular}{lcccc}
\hline & Treatment 1 & Treatment 2 & Treatment 3 & Treatment 4 \\
\hline Nurse 1 & 20 & 20 & & \\
Nurse 2 & 20 & & 20 & \\
Nurse 3 & 20 & & & 30 \\
\hline
\end{tabular}

There are eight patients, in a working day, who need domiciliary visits by the health professionals. The information regarding the treatments for each patient is described in Table 2.

TABLE 2. Treatments needed for each patient in the case study.

\begin{tabular}{lcccc}
\hline & Treatment 1 & Treatment 2 & Treatment 3 & Treatment 4 \\
\hline Patient 1 & $\mathrm{X}$ & & & \\
Patient 2 & $\mathrm{X}$ & $\mathrm{X}$ & & \\
Patient 3 & $\mathrm{X}$ & & $\mathrm{X}$ & \\
Patient 4 & & $\mathrm{X}$ & $\mathrm{X}$ & \\
Patient 5 & $\mathrm{X}$ & & & $\mathrm{X}$ \\
Patient 6 & $\mathrm{X}$ & & & $\mathrm{X}$ \\
Patient 7 & & & & \\
Patient 8 & & & \\
\hline
\end{tabular}

The eight patients live in four different locations. Therefore, each nurse needs to move from the Health Center (the origin) to the patient's home to provide the health care. Patients 1 and 2 live in Location 1, patients 3, 4 and 5 live in Location 2 and patients 6 and 7 live in Location 3; finally, the patient 8 lives in Location 4.

It is also important to note that the locations of the patients have different travel distances. Table 3 describes the time of travel between different locations. A time of 15 minutes was set to go from a house to another in the same location.

TABLE 3. Information about the time spent (min) in the travel between different locations in the case study.

\begin{tabular}{lcccc}
\hline & Location 1 & Location 2 & Location 3 & Location 4 \\
\hline Location 1 & 15 & 40 & 45 & 50 \\
Location 2 & 40 & 15 & 60 & 65 \\
Location 3 & 45 & 60 & 15 & 65 \\
Location 4 & 50 & 65 & 65 & 15 \\
\hline
\end{tabular}

Based on all the data mentioned above, the objective is to do the nurses schedule, in order to minimize the total time needed to provide all the treatments to all the patients and return to the Health Centers.

This problem was solved using the genetic algorithm method described in the next section.

\section{GENETIC ALGORITHM}

To solve the scheduling problem of the nurses routes the Genetic Algorithm was used.

The Genetic algorithm (GA) was originally proposed by John Holland [5]. GA is a stochastic method, whose mechanism is based on simplifications of an evolutionary processes observed in nature, namely selection, mutation and crossover $[6,7,8,9]$. As opposed to many other optimization methods, genetic algorithm works with a population 
of solutions instead of one single solution. In GA, the solutions are combined to generate new ones until a satisfactory solution is obtained, i.e. until the stop criteria is met. In this work it were used the genetic algorithm presented in MatLab Optimization toolbox [10] and the genetic algorithm version presented in the work [6].

\section{RESULTS AND DISCUSSION}

The numerical results were obtained using an Intel(R) Core(TM) i7 CPU 2.2GHz with 6.0 GB of RAM.

Since the genetic algorithm is a stochastic method, each implementation was tested with 50, 100 and 200 runs, in order to evaluate the results. Table 4 presents the percentage of successful runs of each implementation of the genetic algorithm: GA 1 is the implementation of the genetic algorithm of the MatLab Optimization Toolbox and GA 2 is the genetic algorithm according to work [6].

TABLE 4. Successful runs of GA implementations

\begin{tabular}{lccc}
\hline & 50 runs & 100 runs & 200 runs \\
\hline GA 1 & $0 \%$ & $5 \%$ & $10 \%$ \\
GA 2 & $100 \%$ & $100 \%$ & $100 \%$ \\
\hline
\end{tabular}

The execution time average of the successful runs for GA versions is as follows:: GA 1 take 5.64 seconds while and the GA 2 needs 22.2 seconds to solve the optimization problem.

Analyzing the numerical results, it is possible to see that the implementation of GA present in the MatLab Optimization toolbox rarely identifies the optimal solution of the problem. In many runs the solution is not feasible. In opposition, GA 2 is $100 \%$ successful since it finds a feasible solution in all runs.

Figure 1 depicts graphically the obtained solution.

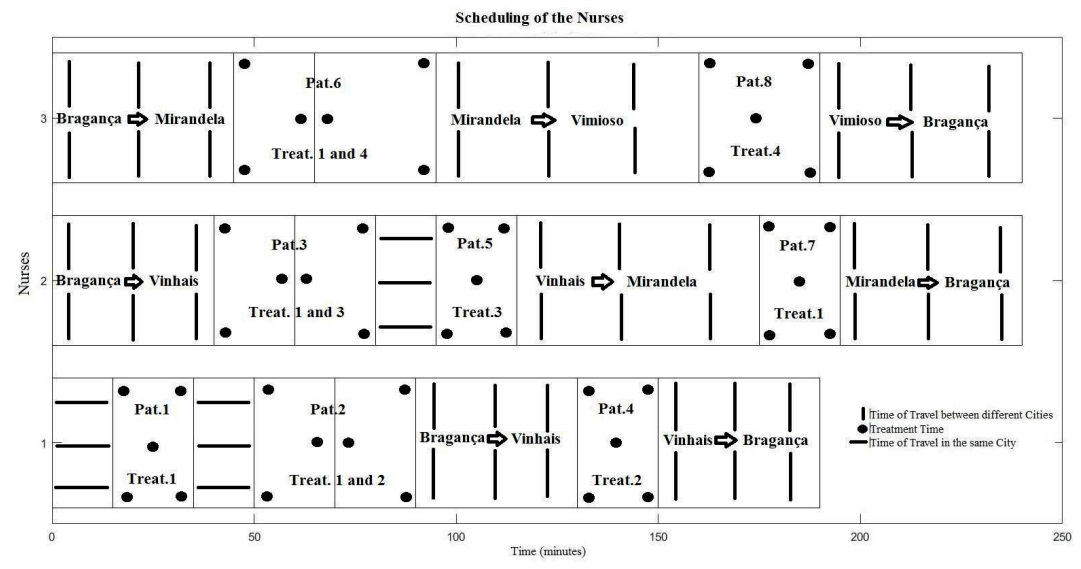

FIGURE 1. Optimal schedule for the case study.

The time needed to each nurse perform care treatment is represented by points. The vertical lines, show the time of travel between different locations. It should be noted that has been assigned 15 minutes ride between to different houses in the same city. For example, the nurse 1 starts its care to a patient in Bragança, needs 15 minutes, in the same city, to go visit another patient (this situation is represented by the horizontal lines).

Analyzing Figure 1 it is possible to see that the minimum time needed for the health team to perform all the visits and return to the Health Center is 240 minutes.

\section{CONCLUSIONS AND FUTURE WORK}

Optimization can be used very advantageously in the context of Health Centers scheduling for home care of the aged people. The scheduling problem of nurses teams was efficiently solved using the genetic algorithm. Moreover the optimal solution was found quite fast. 
This approach represents a gain for all the involved people, health professionals and patients.

For future work, the complexity of the problem could be increased, for instance, increasing the number of nurses, patients or treatments. Another approach is to reformulate the problem and take into account the number of cars available in the Health Center.

\section{ACKNOWLEDGMENTS}

This work has been supported by COMPETE: POCI-01-0145-FEDER-007043 and FCT - Fundação para a Ciência e Tecnologia within the Project Scope: UID/CEC/00319/2013.

\section{REFERENCES}

1. S. Nickel, M. Schröder and Jörg Steeg, Eur. J. Oper. Res., pp.574-587 (2011).

2. E. Benzarti, E. Sahin and Y. Dallery, Decis. Support Syst., 55, pp. 587-598 (2012)

3. S. Bertels and T. Fahle, Comput. Oper. Res., 33, pp.2866-2890 (2006).

4. M. S. Rasmussen, T. Justesen, A. Dohn and J. Larsen, Eur. J. Oper. Res., 219, pp. 598-610 (2012).

5. J.H. Holland, Adaptation in natural and artificial systems, University of Michigan Press, Ann Arbor, 1975.

6. A. Curralo, A. I. Pereira, J. Barbosa and P. Leitão, "Sensibility Study in a Flexible Job Shop Scheduling Problem, Numerical Analysis and Applied Mathematics, Book Series: AIP Conference Proceedings, 2013.

7. D. Goldberg, Genetic Algorithms in Search, Optimization \& Machine Learning, Addison-Wesley, 1989.

8. M. Kumar, M. Husian, N. Upreti and D. Gupta, Genetic Algorithm: Review and Application, International Journal of Information Technology and Knowledge Management, pp. 451-454 (2010).

9. S. Singiresu, Engineering Optimization: Theory and Practice, John Wiley \& Sons, Inc., New Jersey, 2009.

10. MathWork, www.mathworks.com, 2016. 